\title{
Efeito da suplementação com óleo de avocado (Persea americana Mill) na temperatura superficial corpórea de equinos antes e após exercício em esteira
}

[Effect of supplementation with avocado oil (Persea americana Mill) on body surface temperature of horses before and after exercise on treadmill]

M.G. Carvalho, T.Y M. Akutagawa, T.Y. Nitta, N.M.G. Mazzante, J.R.B. Silva,

B.P. Santarosa, C.A. Rodrigues, C.A. Hussni, A.L.G. Alves, M.J. Watanabe

Faculdade de Medicina Veterinária e Zootecnia - Universidade Estadual Paulista (FMVZ-UNESP) - Botucatu, SP

\section{RESUMO}

Oito equinos foram distribuídos em delineamento randomizado cruzado, sendo um grupo sem suplementação (GC) e outro grupo suplementado com óleo de avocado (GOAv) por um período de sete semanas. Ao fim da sexta semana, os animais foram submetidos a teste padrão de exercício progressivo (TPEP) e, após sete dias, a teste de baixa intensidade e longa duração (BILD). Após o primeiro ciclo, houve período de descanso "washout" de 30 dias para troca de grupos para o segundo ciclo, que seguiu o protocolo do primeiro. A termorregulação foi avaliada com base na temperatura retal e na temperatura superficial corpórea, obtidas por termografia, de 15 regiões de interesse. A temperatura retal e as imagens termográficas foram obtidas antes, um minuto e 15 minutos após o exercício. Não houve diferença entre os grupos GC e GOAv em nenhum momento. Os resultados obtidos neste estudo revelaram que a suplementação de 5\% da matéria seca (MS) com óleo de avocado por seis e sete semanas não influenciou na termorregulação com base na temperatura superficial corpórea dos equinos submetidos ao teste padrão de exercício progressivo (TPEP) e ao exercício de baixa intensidade e longa duração (BILD), respectivamente.

Palavras-chave: abacate, metabolismo lipídico, suplemento, termografia, termorregulação

\begin{abstract}
Eight equines were distributed in a randomized crossover design, one control group (CG) without supplementation and another group supplemented $(S G)$ with avocado oil for a period of six weeks. At the end of the sixth week, the animals were submitted to standard exercise test (SET) and after seven days to the low intensity test (LIT). After the first cycle, there was a 30-day washout rest period to exchange groups for the second cycle, which followed the protocol of the first one. Thermoregulation was evaluated based on rectal temperature and body surface temperature of 15 regions of interest obtained by thermography. Rectal temperature and thermographic images were obtained before, one minute and 15 minutes after exercise. There was no difference between the CG and SG at any time. The results obtained in this study revealed that the supplementation of 5\% of dry matter with avocado oil for six and seven weeks did not influence the thermoregulation based on the body surface temperature of the horses submitted to SET and LIT, respectively.
\end{abstract}

Keywords: avocado, lipid metabolism, supplement, thermography, thermoregulation

\section{INTRODUÇÃO}

A suplementação com óleos vegetais na dieta de cavalos pode favorecer o desempenho atlético pelo aumento de energia disponível e pela redução do incremento calórico, pois a formação

Recebido em 20 de dezembro de 2019

Aceito em 16 de março de 2020

E-mail:mgc.unesp@hotmail.com de ATP (adenosina trifosfato), via ácidos graxos, produz em torno de $3 \%$ a menos de calor quando comparada à oxidação via glicólise (Kohn et al., 1996). Dentre os óleos vegetais, o óleo de avocado tem se destacado por sua composição e ação no organismo. É composto principalmente por ácidos graxos monoinsaturados, sendo o 
oleico o mais abundante; possui ação antioxidante, hepatoprotetora, antitrombogênica e potenciais benefícios à saúde cardiovascular. Além disso, requer menor gasto energético para sua digestão, fornecendo mais energia para o animal, sem risco de sobrecarga pelo aumento da ingestão de carboidratos e com menor produção de calor metabólico (Dreher e Davenport, 2013; Ranade e Thiagarajan, 2015).

De acordo com Pösö et al. (2004), os cavalos atletas podem ingerir até $20 \%$ do total da necessidade energética diária advinda de lipídeos. Porém, é necessário um período de adaptação do metabolismo equino e das enzimas do trato digestório à suplementação rica em gordura, para aumentar a capacidade de utilização e oxidação dos ácidos graxos como fonte de energia, poupando as reservas de glicogênio. Esse período pode variar de 21 dias a 12 semanas (Oldham et al., 1990; Harking et al., 1992; Scott et al., 1993; Frape, 1994; Lewis, 2000; Dunnett et al., 2002; Pösö et al., 2004; Mattos et al., 2006).

Quanto à possibilidade de toxicidade do óleo do avocado, não há descrições na literatura, tendo somente descrições de intoxicação com a folha da árvore do avocado em equinos (McKenzie e Brown, 1991; Lorbiecki et al., 2013).

Scott et al. (1993) utilizaram oito equinos Puro Sangue Inglês em estudo randomizado cruzado, com dieta prévia para atingir teor moderado de gordura corporal, seguida de dieta para manutenção e adaptação ao exercício, sendo condicionados ao galope por 28 dias para atingir nível consistente de aptidão física. Após esse período, receberam a dieta com suplementação de gordura por 21 dias e, então, os animais realizaram teste de exercício composto por três sprints de $600 \mathrm{~m}$ em pista ovalada. A produção de calor foi estimada por cálculos baseados no gasto de energia durante o trabalho, o qual foi obtido por meio de amostras de volumes de ar expirado coletadas ao final do terceiro sprint, em repouso. Houve redução de $14 \%$ na produção de calor total de equinos alimentados com gordura adicionada à ração, sugerindo que maior quantidade de energia poderia ser utilizada para a atividade física e o armazenamento de glicogênio, em vez da perda na forma de calor.
A termorregulação pode ser avaliada pelo exame termográfico, em que a câmera termográfica capta as ondas eletromagnéticas de frequência infravermelha e as transforma em uma representação pictórica da temperatura superficial corpórea, de acordo com o calor emitido por um corpo (Andrade Filho, 1999; Turner, 1991, 2001). Em exercícios de alta intensidade e curta duração, o organismo produz mais calor do que consegue dissipar, levando ao estresse térmico, restabelecendo troca efetiva após o término do exercício (McCutcheon e Geor, 2008; Hodgson, 2014). Já em exercício de baixa intensidade e longa duração, o organismo consegue dissipar o calor conforme vai produzindo, de forma mais equilibrada $\mathrm{e}$ mantendo a temperatura interna estável (Nadel, 1988). Diante da relevância do estudo da fisiologia do cavalo atleta e dos possíveis suplementos para melhoria de desempenho, este trabalho teve como objetivo avaliar a influência da suplementação com óleo de avocado na temperatura superficial corpórea, obtida por termografia, de equinos da raça Puro Sangue Árabe (PSA) destreinados e submetidos a teste padrão de exercício progressivo (TPEP).

\section{MATERIAL E MÉTODOS}

Este estudo foi aprovado pela Comissão de Ética no Uso de Animais (Ceua) da Faculdade de Medicina Veterinária e Zootecnia (FMVZ), Universidade Estadual Paulista (Unesp), câmpus de Botucatu, protocolo n`165/2016 - Ceua.

Foram utilizados oito equinos da raça Puro Sangue Árabe, machos castrados, hígidos, com massa corporal média de $341 \pm 15 \mathrm{~kg}$ e idade de $5 \pm 1$ anos, desferrados, ao menos há três meses sem atividade física controlada e pertencentes ao plantel de equinos da FMVZ/Unesp, Botucatu. Os animais foram selecionados após serem submetidos a exame físico e a exame específico dos sistemas locomotor, respiratório e cardiocirculatório, além de exames laboratoriais, como hemograma, leucograma e perfil bioquímico sérico.

O manejo nutricional foi estabelecido para atender as necessidades nutricionais de cavalos em manutenção (NRC, 2007), resultando na ingestão de matéria seca de $2 \%$ do peso vivo (PV), em uma relação de volumoso/concentrado de $1,25 / 0,75 \%$, respectivamente, sendo composta 
de feno de capim coast-cross (cultivar Cynodon dactylon) e concentrado comercial para equinos (Proequi 13 laminados ${ }^{\circledR}-$ Guabi Ind. Brasileira, Brasil) fornecidos em duas refeições, às sete horas e às 17h30, em cochos individuais. O suplemento mineral (Coequi Plus ${ }^{\circledR}$ - Tortuga Cia. Zootécnica Agrária, Brasil) e a água foram oferecidos ad libitum.
Este estudo consistiu de delineamento randomizado cruzado (Fig. 1), em que oito equinos da raça Árabe foram divididos aleatoriamente em dois grupos de quatro animais, que passaram por dois tratamentos por ciclo (suplementados com óleo de avocado GOAv e controle - GC), sendo distribuídos quatro animais por tratamento/ciclo. Foram realizados dois ciclos com 30 dias de intervalo, visando diminuir a influência dos tratamentos.

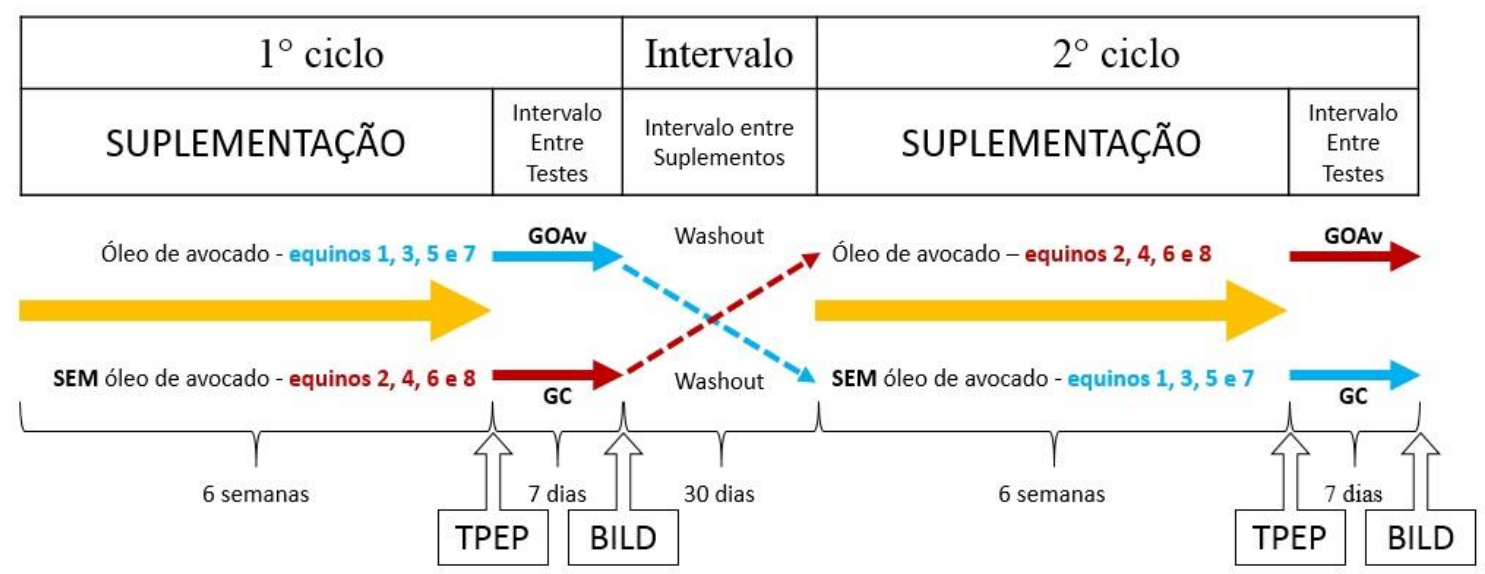

Figura 1. Distribuição dos oito equinos nos grupos, etapas de suplementação e testes físicos de exercício em esteira.

Os grupos foram divididos como: grupo óleo de avocado (GOAv, $\mathrm{n}=8) \quad-\quad$ equinos suplementados, que receberam diariamente $5 \%$ de óleo de avocado referente à matéria seca da dieta por $\mathrm{kg}$ antes do teste padrão de exercício progressivo (TPEP) ou do teste de exercício de baixa intensidade e longa duração (BILD); grupo controle $(\mathrm{GC}, \mathrm{n}=8)$ - equinos, que não receberam suplementação com óleo antes do teste padrão de exercício progressivo (TPEP) ou do teste de exercício de baixa intensidade e longa duração (BILD).

Antes da realização dos testes, todos os equinos passaram por fase de condicionamento, que consistiu de período de 30 dias, visando à padronização das respostas fisiológicas e metabólicas dos cavalos durante o experimento. Os cavalos foram submetidos à adaptação ao manejo nutricional, ao ambiente de realização dos testes físicos (manipulação no tronco, instrumentação e equipe) e à realização de exercício físico em esteira nos aspectos do exercício na esteira (inclinação da esteira, mudança de velocidade e alta velocidade). A suplementação com óleo de avocado (Jaguacy Avocado Brasil ${ }^{\circledR}$ ) foi realizada diariamente, por meio do cálculo do volume de óleo correspondente a $5 \%$ da matéria seca da dieta, sendo o volume, em média $600 \mathrm{~g}$, dividido em duas porções ao dia $(300 \mathrm{~g})$, misturado à ração e, então, oferecido em cochos individuais.

A suplementação foi realizada pelo período de sete semanas no total. No período de adaptação, correspondente aos sete primeiros dias, os cavalos receberam $50 \%$ da dose. O grupo controle recebeu somente a ração comercial para equinos, também em cochos individuais. $\mathrm{O}$ protocolo do teste padrão de exercício progressivo (TPEP) foi realizado com a esteira inclinada a $6 \%$, com fases sequenciais de velocidades progressivas (intensidades de exercício), de acordo com o protocolo: velocidade inicial de $1,8 \mathrm{~m} / \mathrm{s}$ por um período de cinco minutos, seguindo a $4,0 \mathrm{~m} / \mathrm{s}$ por três minutos, 6,$0 ; 7,0 ; 8,0 ; 9,0$ e $10,0 \mathrm{~m} / \mathrm{s}$ por dois minutos em cada velocidade, ou até quando os cavalos não conseguissem manter o galope, mesmo sendo estimulados. 
Já o protocolo do teste de exercício de baixa intensidade e longa duração (BILD) consistiu da inclinação da esteira a $6 \%$ e da velocidade de exercício correspondente à $\mathrm{V}_{140}$ de cada animal, obtida no TPEP, pelo período de $60 \mathrm{~min}$, e, na sequência, $10 \mathrm{~min}$ de desaquecimento ativo a $1,8 \mathrm{~m} / \mathrm{s}$, com $0 \%$ de inclinação. A $\mathrm{V}_{140}$ corresponde à velocidade do animal quando sua frequência cardíaca atinge $140 \mathrm{bpm}$, obtida por uma regressão linear de acordo com os batimentos cardíacos mensurados no TPEP.

O exame termográfico seguiu as recomendações de Basile et al. (2010), sendo os animais ambientados 90min antes do exercício, às três horas da manhã, evitando-se a interferência da luz solar sobre a pele. Os cavalos tiveram os cascos e a pele limpos, com o uso de limpador de casco, escova e compressa de algodão, sem utilização de água. Após o preparo dos animais, o Centro de Medicina Equina Esportiva permaneceu com as portas fechadas e os cavalos não foram manipulados desde 30min anteriores à avaliação. A temperatura e a umidade do ar foram mensuradas com termo-higrômetro digital portátil e registradas em cada um dos momentos.

Três imagens termográficas foram capturadas, para análise de 15 regiões cutâneas, em três momentos: pré (M0), um minuto (M1) e 15 (M15) minutos após o exercício, com os cavalos em estação. Todas as imagens foram obtidas no mesmo ambiente, utilizando-se termógrafo (FLIR SC660 ${ }^{\circledR}$ ) ajustado com emissividade de 0,98 e calibrado, ilustrando o contraste da banda coronária do casco do equino representada em vermelho (vista dorsal), na paleta de cores/temperatura rainbow, com intervalo entre as temperaturas máxima e mínima de $10^{\circ} \mathrm{C}$, de acordo com o preconizado por Basile et al. (2010). As imagens termográficas foram obtidas na seguinte ordem: face dorsal da região distal dos membros torácicos, região lateral esquerda da cabeça e face caudal da região da musculatura dos membros pélvicos, sempre a $1,5 \mathrm{~m}$ de distância da região escolhida, com a superfície da lente posicionada paralelamente à superfície a ser examinada, conforme preconizado por Basile et al. (2010). A imagem dos membros torácicos foi realizada com o foco entre os ossos metacarpais terceiros, e a avaliação termográfica foi feita em cinco regiões anatômicas de cada membro (Fig. 2).

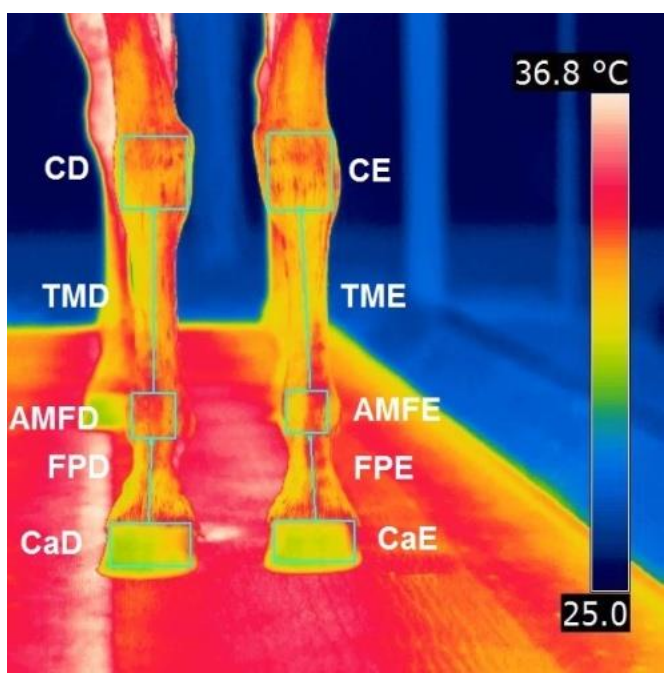

Figura 2. Termograma da face dorsal da região distal dos membros torácicos. As regiões cutâneas mensuradas foram o aspecto dorsal de: CD - carpo direito; CE - carpo esquerdo; TMD - terceiro metacarpal direito; TME - terceiro metacarpal esquerdo; AMFD - articulação metacarpofalângica direita; AMFE - articulação metacarpofalângica; FPD - falange proximal direita; FPE - falange proximal esquerda; $\mathrm{CaD}$ casco direito; $\mathrm{CaE}$ - casco esquerdo.

$\mathrm{Na}$ região lateral esquerda da cabeça, estabeleceu-se a região do globo ocular, restringindo-se à análise da borda palpebral posteromedial da pálpebra inferior e à carúncula lacrimal, onde foi obtido o ponto máximo da temperatura (Fig. 3A). Nos membros pélvicos, escolheram-se as regiões do músculo semitendíneo e da porção cranial do músculo bíceps femoral (Fig. 3B). As imagens foram analisadas por meio do software Flir QuickReport $^{\circledR}$ 1.2 SP2, em que se utilizou a temperatura máxima de cada região anatômica. A temperatura retal foi aferida com termômetro clínico digital flexível G-Tech ${ }^{\circledR}$, em todos os momentos. 


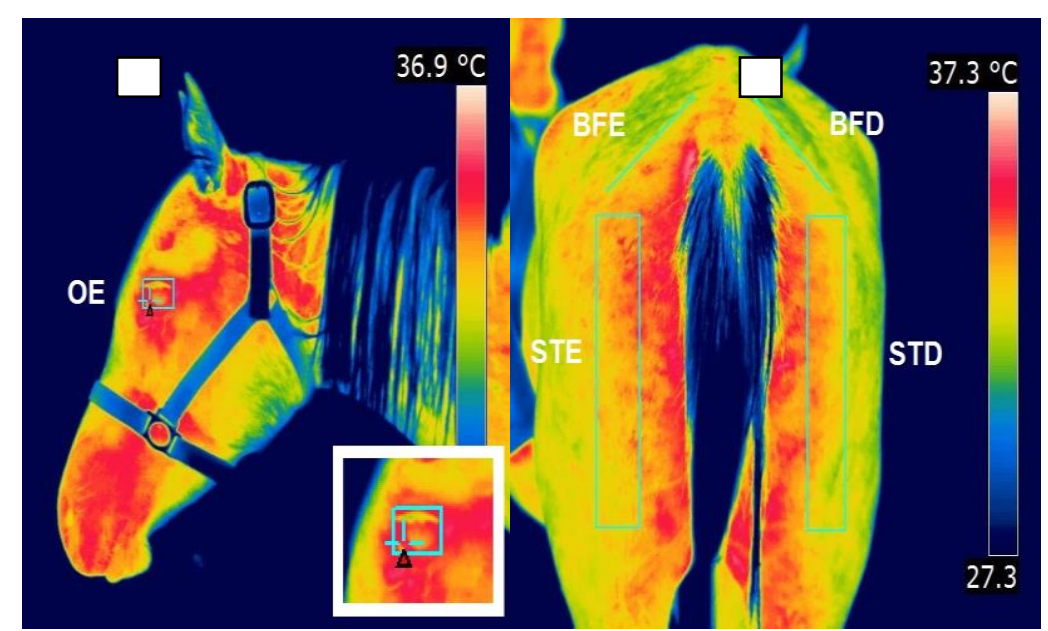

Figura 3. A) Termograma da cabeça lateral esquerda, com delimitação do olho esquerdo (OE) para análise da borda palpebral posteromedial da pálpebra inferior, onde foi obtido o ponto máximo (seta) da temperatura ocular. B) Termograma da musculatura pélvica, das regiões cutâneas de: STE - músculo semitendíneo esquerdo; STD - músculo semitendíneo direito; BFE - músculo bíceps femoral esquerdo; $\mathrm{BFD}$ - músculo bíceps femoral direito.

Os dados obtidos por termografia foram padronizados em função da temperatura ambiente no dia da primeira avaliação, conforme descrito no estudo de Basile et al. (2010), para possibilitar a comparação entre essas temperaturas ao longo do tempo. Os dados apresentaram distribuição normal pelo teste de Kolmogorov-Smirnov. Para cada região corpórea, diferenças de média entre tratamentos e momentos foram avaliadas por meio de análise de variância (ANOVA), seguida de teste post hoc de Tukey. Com o intuito de controlar efeitos confundidores nas análises, os modelos de ANOVA incluíram a temperatura ambiental como covariável e a animal como efeito fixo. Um nível de significância de $\mathrm{P}<0,05$ foi adotado para todas as comparações. Todas as análises foram realizadas com auxílio do software $\mathrm{R}$ v3.4.4 (R Core..., 2018).

Padronizaram-se os dados obtidos pela termografia de acordo com a temperatura ambiente, do Centro de Medicina Equina Esportiva, do primeiro dia de teste, que foi $24,3^{\circ} \mathrm{C}$, conforme descrito no estudo de Basile et al. (2010), para possibilitar a comparação entre as temperaturas ao longo do tempo.

\section{RESULTADOS E DISCUSSÃO}

Durante todo o experimento, os cavalos não apresentaram redução na ingestão de alimento devido à suplementação com o óleo de avocado, portanto a palatabilidade não influenciou os animais. Os valores da temperatura ambiental e umidade relativa do ar foram monitorados em todos os momentos no ambiente experimental, variando de 18,9 a $26,5^{\circ} \mathrm{C}$ e de 45 a $99 \%$, respectivamente. Esses dados foram utilizados para prever o comprometimento de rotas respiratórias e evaporativas em climas quentes e úmidos, de acordo com método descrito por Cymbaluk e Christison (1990), em que se soma a temperatura ambiente $\left({ }^{\circ} \mathrm{F}\right)$ à umidade relativa. Os valores apresentaram-se entre 119 e 178, não atingindo o valor de 180, no qual a atividade física não é recomendada devido ao fato de a perda de calor por evaporação ser extremamente difícil.

As outras variáveis ambientais não interferiram na temperatura dos animais, pois os testes eram realizados em ambiente fechado, com ambientação feita uma hora e meia antes do teste, sem interferência de luz solar e sem exposição a chuvas ou ventos. Os dados de temperatura derivados das 15 regiões corpóreas e de temperatura retal dos equinos submetidos ao TPEP e ao BILD estão apresentados nas Tab. 1 e 2 , respectivamente.

Nenhuma variável analisada apresentou diferença entre os grupos GOAv e GC em nenhum dos testes, o que demonstra que não 
houve influência da suplementação com óleo de avocado nas temperaturas superficial corpórea e retal analisadas neste estudo. Apesar de Scott et al. (1993) terem constatado redução de $14 \%$ do calor corporal de equinos alimentados com dietas com adição de óleo, esse valor foi estimado por método diferente do presente estudo, por cálculos baseados no gasto de energia durante o trabalho, obtido por meio de amostras de volumes de ar expirado coletadas ao final do exercício.

Tabela 1. Temperaturas $\left({ }^{\circ} \mathrm{C}\right)$ representadas pela média \pm desvio-padrão (DP) das diferentes regiões anatômicas cutâneas submetidas à termografia e da temperatura retal antes, após um minuto e 15 minutos do teste padrão de exercício progressivo

\begin{tabular}{|c|c|c|c|c|}
\hline \multirow{2}{*}{ Região } & \multirow{2}{*}{ Tratamento } & \multicolumn{3}{|c|}{ Momentos } \\
\hline & & M0 & M1 & M15 \\
\hline \multirow{2}{*}{ RET } & GOAv & $37,05 \pm 0,25^{\mathrm{Ab}}$ & $39,18 \pm 0,59^{\mathrm{Aa}}$ & $39,54 \pm 0,48^{\mathrm{Aa}}$ \\
\hline & $\mathrm{GC}$ & $37,18 \pm 0,36^{\mathrm{Ab}}$ & $39.33 \pm 0,46^{\mathrm{Aa}}$ & $39.36 \pm 0,39^{\text {Аa }}$ \\
\hline \multirow{2}{*}{$\mathrm{OE}$} & GOAv & $35,30 \pm 0,27^{\mathrm{Ab}}$ & $37,84 \pm 0,52^{\mathrm{Aa}}$ & $37,16 \pm 0,80^{\mathrm{Aa}}$ \\
\hline & GC & $35,34 \pm 0,31^{\mathrm{Ac}}$ & $37,95 \pm 0,49^{\mathrm{Aa}}$ & $37,41 \pm 0,25^{\mathrm{Ab}}$ \\
\hline \multirow{2}{*}{ CD } & GOAv & $31,57 \pm 2,56^{\mathrm{Ab}}$ & $36,47 \pm 1,47^{\mathrm{Aa}}$ & $35,94 \pm 1,42^{\mathrm{Aa}}$ \\
\hline & GC & $30,06 \pm 3,03^{\mathrm{Ab}}$ & $36,32 \pm 1,19^{\mathrm{Aa}}$ & $35,82 \pm 1,18^{\mathrm{Aa}}$ \\
\hline \multirow{2}{*}{$\mathrm{CE}$} & GOAv & $31,26 \pm 2,56^{\mathrm{Ab}}$ & $36,17 \pm 1,47^{\mathrm{Aa}}$ & $35,83 \pm 1,28^{\mathrm{Aa}}$ \\
\hline & GC & $30,37 \pm 2,68^{\mathrm{Ab}}$ & $36,42 \pm 1,58^{\mathrm{Aa}}$ & $36,11 \pm 1,55^{\mathrm{Aa}}$ \\
\hline \multirow{2}{*}{ TMD } & GOAv & $31,05 \pm 2,39^{\mathrm{Ab}}$ & $34,52 \pm 1,27^{\mathrm{Aa}}$ & $34,96 \pm 1,76^{\mathrm{Aa}}$ \\
\hline & $\mathrm{GC}$ & $29,40 \pm 3,09^{\mathrm{Ab}}$ & $34,40 \pm 0,69^{\mathrm{Aa}}$ & $34,65 \pm 1,38^{\mathrm{Aa}}$ \\
\hline \multirow{2}{*}{ TME } & GOAv & $30,69 \pm 2,28^{\mathrm{Ab}}$ & $34,68 \pm 1,32^{\mathrm{Aa}}$ & $35,11 \pm 1,63^{\mathrm{Aa}}$ \\
\hline & GC & $29,47 \pm 2,86^{\mathrm{Ab}}$ & $34,66 \pm 0,78^{\mathrm{Aa}}$ & $34,81 \pm 1,18^{\mathrm{Aa}}$ \\
\hline \multirow{2}{*}{ AMFD } & GOAv & $32,66 \pm 2.48^{\mathrm{Ab}}$ & $35,25 \pm 1,30^{\mathrm{Aa}}$ & $35,80 \pm 1,98^{\text {Аа }}$ \\
\hline & GC & $30,25 \pm 3,66^{\mathrm{Ab}}$ & $35,88 \pm 1,42^{\mathrm{Aa}}$ & $35,52 \pm 1,47^{\mathrm{Aa}}$ \\
\hline \multirow{2}{*}{ AMFE } & GOAv & $32,43 \pm 2,62^{\mathrm{Ab}}$ & $35,90 \pm 1,41^{\mathrm{Aa}}$ & $35,86 \pm 1,94^{\mathrm{Aa}}$ \\
\hline & GC & $30,17 \pm 3,11^{\mathrm{Ab}}$ & $36,16 \pm 1,42^{\mathrm{Aa}}$ & $35,85 \pm 0,94^{\mathrm{Aa}}$ \\
\hline \multirow{2}{*}{ FPD } & GOAv & $31,82 \pm 1,45^{\mathrm{Ab}}$ & $33,65 \pm 1,60^{\mathrm{Aab}}$ & $34,82 \pm 1,58^{\mathrm{Aa}}$ \\
\hline & GC & $29,58 \pm 2,97^{\mathrm{Ab}}$ & $33,87 \pm 1,51^{\mathrm{Aa}}$ & $33,67 \pm 1,73^{\mathrm{Aa}}$ \\
\hline \multirow{2}{*}{ FPE } & GOAv & $32,00 \pm 1,86^{\mathrm{Ab}}$ & $33,93 \pm 1,70^{\mathrm{Aab}}$ & $34,36 \pm 1,65^{\mathrm{Aa}}$ \\
\hline & GC & $30,25 \pm 1,92^{\mathrm{Ab}}$ & $33,85 \pm 1,74^{\mathrm{Aa}}$ & $34,49 \pm 1,45^{\mathrm{Aa}}$ \\
\hline \multirow{2}{*}{$\mathrm{CaD}$} & GOAv & $34,54 \pm 1,75^{\mathrm{Ab}}$ & $35,68 \pm 0.93^{\mathrm{Aab}}$ & $36,53 \pm 1,52^{\mathrm{Aa}}$ \\
\hline & GC & $31,89 \pm 4,21^{\mathrm{Ab}}$ & $35,11 \pm 2,00^{\mathrm{Aab}}$ & $36,02 \pm 1,59^{\mathrm{Aa}}$ \\
\hline \multirow{2}{*}{$\mathrm{CaE}$} & GOAv & $34,46 \pm 1,85^{\mathrm{Ab}}$ & $35,70 \pm 0,97^{\mathrm{Aab}}$ & $36,41 \pm 1,44^{\mathrm{Aa}}$ \\
\hline & GC & $32,61 \pm 3,01^{\mathrm{Ab}}$ & $35,37 \pm 2,10^{\mathrm{Aab}}$ & $36,41 \pm 1,82^{\mathrm{Aa}}$ \\
\hline \multirow{2}{*}{ STD } & GOAv & $34,77 \pm 1,64^{\mathrm{Ab}}$ & $38,95 \pm 1,40^{\mathrm{Aa}}$ & $37,88 \pm 1,25^{\mathrm{Aa}}$ \\
\hline & GC & $34,23 \pm 1,53^{\mathrm{Ab}}$ & $38,34 \pm 1,48^{\mathrm{Aa}}$ & $37,59 \pm 1,30^{\mathrm{Aa}}$ \\
\hline \multirow{2}{*}{ STE } & GOAv & $34,76 \pm 1,16^{\mathrm{Ab}}$ & $39,09 \pm 1,39^{\mathrm{Aa}}$ & $38,12 \pm 1,31^{\mathrm{Aa}}$ \\
\hline & GC & $34,58 \pm 1,65^{\mathrm{Ab}}$ & $38,43 \pm 1,57^{\mathrm{Aa}}$ & $37,68 \pm 1,41^{\mathrm{Aa}}$ \\
\hline \multirow{2}{*}{ BFD } & GOAv & $32,81 \pm 1,55^{\mathrm{Ab}}$ & $37,01 \pm 1,42^{\mathrm{Aa}}$ & $35,86 \pm 1,07^{\mathrm{Aa}}$ \\
\hline & GC & $32,35 \pm 1,16^{\mathrm{Ab}}$ & $36,80 \pm 1,14^{\mathrm{Aa}}$ & $35,88 \pm 1,02^{\mathrm{Aa}}$ \\
\hline \multirow{2}{*}{ BFE } & GOAv & $33,09 \pm 1,51^{\mathrm{Ab}}$ & $36,91 \pm 1,02^{\mathrm{Aa}}$ & $35,92 \pm 1,19^{\text {Аа }}$ \\
\hline & GC & $32,26 \pm 1,29^{\mathrm{Ab}}$ & $37,00 \pm 1,22^{\mathrm{Aa}}$ & $35,89 \pm 0,98^{\mathrm{Aa}}$ \\
\hline
\end{tabular}

Médias com letras maiúsculas distintas nas linhas indicam diferenças significativas entre grupos.

Médias com letras minúsculas distintas nas colunas indicam diferenças significativas entre momentos.

RET - retal; CD - carpo direito; CE - carpo esquerdo; TMD - terceiro metacarpal direito; TME - terceiro metacarpal esquerdo; AMFD - articulação metacarpofalângica direita; AMFE - articulação metacarpofalângica esquerda; FPD falange proximal direita; $\mathrm{FPE}$ - falange proximal esquerda; $\mathrm{CaD}$ - casco direito; $\mathrm{CaE}$ - casco esquerdo; $\mathrm{OE}$ - olho esquerdo; STE - músculo semitendíneo esquerdo; STD - músculo semitendíneo direito; BFE - músculo bíceps femoral esquerdo; BFD - músculo bíceps femoral direito.

GOAv: grupo suplementado com 5\% de óleo de avocado; GC: grupo controle. 
Efeito da suplementação...

Tabela 2. Temperaturas $\left({ }^{\circ} \mathrm{C}\right)$ representadas pela média \pm desvio-padrão (DP) das diferentes regiões anatômicas cutâneas submetidas à termografia e da temperatura retal antes, após um minuto e 15 minutos do teste de baixa intensidade e longa duração

\begin{tabular}{|c|c|c|c|c|}
\hline \multirow{2}{*}{ Região } & \multirow{2}{*}{ Tratamento } & \multicolumn{3}{|c|}{ Momentos } \\
\hline & & M0 & M1 & M15 \\
\hline \multirow{2}{*}{ RET } & GOAv & $37,43 \pm 0,37^{\mathrm{Ab}}$ & $39,76 \pm 0,99^{\mathrm{Aa}}$ & $38,93 \pm 0,70^{\mathrm{Aa}}$ \\
\hline & $\mathrm{GC}$ & $37,44 \pm 0,18^{\mathrm{Ac}}$ & $39,87 \pm 0,74^{\mathrm{Aa}}$ & $38,61 \pm 0,81^{\mathrm{Ab}}$ \\
\hline \multirow{2}{*}{$\mathrm{OE}$} & GOAv & $35,24 \pm 0,38^{\mathrm{Ab}}$ & $36,48 \pm 0,81^{\mathrm{Aa}}$ & $36,25 \pm 0,89^{\mathrm{Aa}}$ \\
\hline & GC & $35,09 \pm 0,48^{\mathrm{Ab}}$ & $36,66 \pm 1,00^{\mathrm{Aa}}$ & $36,01 \pm 0,70^{\mathrm{Aab}}$ \\
\hline \multirow{2}{*}{ CD } & GOAv & $30,51 \pm 3,24^{\mathrm{Ab}}$ & $34,56 \pm 1,48^{\mathrm{Aa}}$ & $33,97 \pm 1,12^{\mathrm{Aa}}$ \\
\hline & $\mathrm{GC}$ & $29,05 \pm 2,75^{\mathrm{Ab}}$ & $34,64 \pm 1,41^{\mathrm{Aa}}$ & $34,13 \pm 1,50^{\mathrm{Aa}}$ \\
\hline \multirow{2}{*}{ CE } & GOAv & $29,92 \pm 3,74^{\mathrm{Ab}}$ & $34,67 \pm 1,16^{\mathrm{Aa}}$ & $34,07 \pm 0,52^{\mathrm{Aa}}$ \\
\hline & GC & $28,81 \pm 2,15^{\mathrm{Ab}}$ & $34,76 \pm 2,45^{\text {Аa }}$ & $34,41 \pm 2,08^{\mathrm{Aa}}$ \\
\hline \multirow{2}{*}{ TMD } & GOAv & $29,80 \pm 2,01^{\mathrm{Ab}}$ & $33,50 \pm 1,52^{\mathrm{Aa}}$ & $32,81 \pm 1,58^{\mathrm{Aa}}$ \\
\hline & $\mathrm{GC}$ & $28,35 \pm 2,29^{\mathrm{Ab}}$ & $33,65 \pm 1,93^{\mathrm{Aa}}$ & $32,68 \pm 1,60^{\mathrm{Aa}}$ \\
\hline \multirow{2}{*}{ TME } & GOAv & $29,29 \pm 3,09^{\mathrm{Ab}}$ & $34,22 \pm 1,65^{\mathrm{Aa}}$ & $33,17 \pm 1,33^{\mathrm{Aa}}$ \\
\hline & GC & $28,87 \pm 2,30^{\mathrm{Ab}}$ & $33,49 \pm 1,68^{\mathrm{Aa}}$ & $33,11 \pm 1,98^{\mathrm{Aa}}$ \\
\hline \multirow{2}{*}{ AMFD } & GOAv & $31,29 \pm 2,08^{\mathrm{Ab}}$ & $34,40 \pm 1,24^{\mathrm{Aa}}$ & $33,92 \pm 1,28^{\mathrm{Aa}}$ \\
\hline & GC & $30,11 \pm 3,30^{\mathrm{Ab}}$ & $34,54 \pm 2,07^{\mathrm{Aa}}$ & $34,13 \pm 2,46^{\mathrm{Aa}}$ \\
\hline \multirow{2}{*}{ AMFE } & GOAv & $30,05 \pm 3,06^{\mathrm{Ab}}$ & $34,86 \pm 1,07^{\mathrm{Aa}}$ & $33,82 \pm 1,00^{\mathrm{Aa}}$ \\
\hline & GC & $30,46 \pm 3,59^{\mathrm{Ab}}$ & $34,81 \pm 2,07^{\mathrm{Aa}}$ & $34,34 \pm 1,97^{\mathrm{Aa}}$ \\
\hline \multirow{2}{*}{ FPD } & GOAv & $33,38 \pm 1,58^{\mathrm{Aa}}$ & $33,22 \pm 1,21^{\mathrm{Aa}}$ & $32,93 \pm 0,95^{\mathrm{Aa}}$ \\
\hline & $\mathrm{GC}$ & $31,36 \pm 4,05^{\mathrm{Aa}}$ & $33,37 \pm 1,81^{\mathrm{Aa}}$ & $32,84 \pm 2,39^{\mathrm{Aa}}$ \\
\hline \multirow{2}{*}{ FPE } & GOAv & $32,19 \pm 3,75^{\text {Аа }}$ & $33,66 \pm 1,44^{\mathrm{Aa}}$ & $33,15 \pm 0,97^{\text {Аа }}$ \\
\hline & $\mathrm{GC}$ & $31,99 \pm 4,23^{\mathrm{Aa}}$ & $33,54 \pm 1,64^{\mathrm{Aa}}$ & $33,10 \pm 2,49^{\mathrm{Aa}}$ \\
\hline \multirow{2}{*}{$\mathrm{CaD}$} & GOAv & $35,15 \pm 2,26^{\mathrm{Aa}}$ & $33,38 \pm 0,45^{\text {Аa }}$ & $33,64 \pm 1,34^{\mathrm{Aa}}$ \\
\hline & $\mathrm{GC}$ & $32,72 \pm 4,10^{\mathrm{Aa}}$ & $33,58 \pm 1,56^{\mathrm{Aa}}$ & $34,03 \pm 2,45^{\mathrm{Aa}}$ \\
\hline \multirow{2}{*}{$\mathrm{CaE}$} & GOAv & $32,95 \pm 4,25^{\mathrm{Aa}}$ & $33,38 \pm 0,68^{\mathrm{Aa}}$ & $34,09 \pm 1,47^{\mathrm{Aa}}$ \\
\hline & GC & $32,95 \pm 4,07^{\mathrm{Aa}}$ & $33,84 \pm 1,60^{\mathrm{Aa}}$ & $34,08 \pm 2,13^{\mathrm{Aa}}$ \\
\hline \multirow{2}{*}{ STD } & GOAv & $35,28 \pm 1,47^{\mathrm{Ab}}$ & $37,13 \pm 1,10^{\mathrm{Aa}}$ & $35,92 \pm 0,68^{\text {Aab }}$ \\
\hline & $\mathrm{GC}$ & $33,93 \pm 1,13^{\mathrm{Ab}}$ & $36,27 \pm 1,10^{\mathrm{Aa}}$ & $35,56 \pm 1,15^{\text {Aab }}$ \\
\hline \multirow{2}{*}{ STE } & GOAv & $35,14 \pm 1,60^{\mathrm{Ab}}$ & $37,18 \pm 0,98^{\mathrm{Aa}}$ & $36,05 \pm 0,73^{\mathrm{Aab}}$ \\
\hline & GC & $34,55 \pm 1,45^{\mathrm{Ab}}$ & $36,75 \pm 1,54^{\mathrm{Aa}}$ & $36,24 \pm 1,66^{\mathrm{Aab}}$ \\
\hline \multirow{2}{*}{ BFD } & GOAv & $32,67 \pm 1,46^{\mathrm{Ab}}$ & $35,30 \pm 0,94^{\mathrm{Aa}}$ & $33,90 \pm 0,69^{\mathrm{Aab}}$ \\
\hline & GC & $32,44 \pm 0,91^{\mathrm{Ab}}$ & $35,10 \pm 1,17^{\mathrm{Aa}}$ & $33,51 \pm 1,31^{\mathrm{Aab}}$ \\
\hline \multirow{2}{*}{ BFE } & GOAv & $32,56 \pm 0,91^{\mathrm{Ab}}$ & $34,76 \pm 1,17^{\mathrm{Aa}}$ & $33,64 \pm 0,76^{\mathrm{Aab}}$ \\
\hline & $\mathrm{GC}$ & $32,30 \pm 0,77^{\mathrm{Ab}}$ & $34,36 \pm 1,29^{\mathrm{Aa}}$ & $33,44 \pm 1,41^{\mathrm{Aab}}$ \\
\hline
\end{tabular}

Médias com letras maiúsculas distintas nas linhas indicam diferenças significativas entre grupos.

Médias com letras minúsculas distintas nas colunas indicam diferenças significativas entre momentos.

RET - retal; CD - carpo direito; CE - carpo esquerdo; TMD - terceiro metacarpal direito; TME - terceiro metacarpal esquerdo; AMFD - articulação metacarpofalângica direita; AMFE - articulação metacarpofalângica esquerda; FPD falange proximal direita; FPE - falange proximal esquerda; $\mathrm{CaD}$ - casco direito; $\mathrm{CaE}$ - casco esquerdo; $\mathrm{OE}$ - olho esquerdo; STE - músculo semitendíneo esquerdo; STD - músculo semitendíneo direito; BFE - músculo bíceps femoral esquerdo; BFD - músculo bíceps femoral direito.

GOAv: grupo suplementado com 5\% de óleo de avocado; GC: grupo controle.

Outro fator importante foi em relação à dose utilizada (5\% da MS) e ao período de adaptação (seis semanas), que foram reduzidos devido ao custo do produto, podendo ter sido insuficientes para otimizar a captação de ácidos graxos, visto que outros autores sugeriram suplementação com óleo referente a $10 \%$ da MS e até 12 semanas de adaptação ao metabolismo lipídico (Oldham et al., 1990; Harking et al., 1992; Scott et al., 1993; Frape, 1994; Lewis, 2000; Dunnett et al., 2002; Pösö et al., 2004; Mattos et al., 2006).

No TPEP, a RET apresentou elevação de M0 $\left(37,05 \pm 0,25^{\circ} \mathrm{C}\right)$ para M1 $\left(39,18 \pm 0,59^{\circ} \mathrm{C}\right)$, mantendo a elevação até M15. Berkman et al. (2011) também constataram elevação da 
temperatura retal em pesquisa com teste de esforço, com elevação de $36,9 \pm 0,18^{\circ} \mathrm{C}$ para $40,1 \pm 0,07^{\circ} \mathrm{C}$ após o exercício. Já no BILD, a temperatura retal (RET) elevou-se de M0 $\left(37,44 \pm 0,18^{\circ} \mathrm{C}\right)$ para $\mathrm{M} 1 \quad\left(39,87 \pm 0,74^{\circ} \mathrm{C}\right)$ e reduziu em $\mathrm{M} 15\left(38,61 \pm 0,18^{\circ} \mathrm{C}\right)$, porém sem voltar aos valores de repouso. Esse aumento em M1 ocorreu porque a temperatura central continua a aumentar nos primeiros minutos de recuperação do exercício máximo, à medida que o calor se redistribiu nos músculos (Hodgson, 2014). O fato de a temperatura retal após o BILD reduzir de M1 para M15 demonstra que os mecanismos termorregulatórios de exercícios prolongados são mais eficientes e tendem ao retorno da temperatura interna estável mais rapidamente que no TPEP (Nadel, 1988).

O parâmetro OE elevou-se em ambos os testes em M1, mantendo a elevação até M15. Essa variável tem sido relacionada ao nível de estresse de equinos durante competições, em conjunto com a frequência cardíaca (Bartolomé et al., 2013; Negro et al., 2018). Soroko et al. (2016) também observaram elevação da OE após exercício de cavalos de corrida e não constataram retorno ao valor basal até duas horas após o exercício. As temperaturas máximas das regiões do membro torácico: $\mathrm{CD}, \mathrm{CE}, \mathrm{TMD}$, TME, AMFD, AMFE se comportaram similarmente à temperatura retal no TPEP e no BILD, elevando-se em M1 e mantendo a elevação em M15, o que demonstra pouca participação dessas regiões na termorregulação após exercício. No TPEP, FPD e FPE também apresentaram o mesmo comportamento.

Já no BILD, FPD e FPE se comportaram da mesma maneira que $\mathrm{CaD}$ e $\mathrm{CaE}$, não apresentando elevação após o exercício, ou seja, permaneceram iguais em todos os momentos, o que corrobora os achados de Berkman et al. (2011) em relação ao comportamento antagonista do casco comparado às demais regiões. Estas regiões conseguiram dissipar o calor conforme sua produção, de forma equilibrada e mantendo a temperatura estável (Nadel, 1988). No TPEP, ocorreu elevação da temperatura dos $\mathrm{CaD}$ e $\mathrm{CaE}$ em M15, e a temperatura de M0 manteve-se em M1, conforme o BILD, porém diferiu em M15. Isso mostra maior produção e consequente armazenamento de calor no TPEP, o qual precisa ser dissipado também através dos cascos, diferentemente do estudo de Berkman et al.
(2011), em que não se observou elevação da temperatura do casco em 20 minutos após exercício progressivo em esteira.

As regiões STE, STD, BFE e BFD da musculatura pélvica apresentaram comportamento similar ao comportamento de RET e OE, elevando-se em M1 e mantendo a elevação em M15. Sala et al. (2012) observaram retorno da temperatura superficial da região dos músculos semitendíneo e semimembranáceo somente duas horas após o exercício. Já no BILD, as temperaturas máximas das regiões STE, STD, BFE, BFD elevaram-se em M1, reduziram e retornaram aos valores de repouso (M0) em M15. Durante o exercício, ocorre maior aporte sanguíneo para a musculatura esquelética, o que justifica o aumento da temperatura em M1, seguido de maior participação desses grupos musculares na dissipação do calor, contribuindo, assim, para a termorregulação e o retorno aos valores de repouso em 15 minutos após o exercício.

\section{CONCLUSÃO}

Os resultados obtidos neste estudo revelaram que a suplementação de $5 \%$ da MS com óleo de avocado, por seis e sete semanas, não influenciou na termorregulação com base na temperatura superficial corpórea dos equinos submetidos ao teste padrão de exercício progressivo (TPEP) e ao exercício de baixa intensidade e longa duração (BILD), respectivamente.

\section{AGRADECIMENTOS}

À Coordenação de Aperfeiçoamento de Pessoal de Nível Superior (Capes), pela bolsa de doutorado concedida; à FMVZ/UnespBotucatu, pela infraestrutura e pelos animais utilizados.

\section{REFERÊNCIAS}

ANDRADE FILHO, A.C. Teletermografia: princípios físicos, fisiológicos e fisiopatológicos da produção da imagem e suas indicações na clínica de dor e reabilitação. Acta Fisiátrica, v.6, p.55-59, 1999. 
BARTOLOMÉ, E.; SÁNCHEZ, M.J.; MOLINA, A. et al. Using eye temperature and heart rate for stress assessment in young horses competing in jumping competitions and its possible influence on sport performance. Animal. v.7, p.2044-2053, 2013.

BASILE, R.C.; BASILE, M.T.; ALBERNAZ, R.M. et al. Guia prático de exames termográficos em equinos. Rev. Bras. Med. Equina, v.6, p.24$28,2010$.

BERKMAN, C.; ALBERNAZ, R.M.; BASILE, R.C. et al. Exercício em esteira não elevou a temperatura do casco de equinos. Ciênc. Rural, v.41, p.1398-1404, 2011.

CYMBALUK, N.F.; CHRISTISON, G.I. Environmental effects on thermoregulation and nutrition of horses. Vet. Clin. N. Am. Equine Pract., v.6, p.355-372, 1990.

DREHER, M.L.; DAVENPORT, A.J. Hass avocado composition and potential health effects. Crit. Rev. Food Sci. Nutr., v.53, p.738-750, 2013.

DUNNETT, C.E.; MARLIN, D.J.; HARRIS, R.C. Effect of dietary lipid on response to exercise: relationship to metabolic adaptation. Equine Vet. J., v.34, p.75-80, 2002.

FRAPE, D.L. Diet and exercise performance in the horse. Proc. Nutr. Soc., v.53, p.189-206, 1994.

HARKING, J.D.; MORRIS, G.S.; TULLEY, R.T.; NELSON, A.G.; KAMERLING, S. G. Effect of added dietary fat on racing performance in Thoroughbred horses. J. Equine Vet. Sci., v.12, p.123-129, 1992.

HODGSON, D.R. Thermoregulation I. In: HODGSON, D.R.; MCGOWAN C.M.; MCKEEVER, K.H. The athletic horse: principles and practice of equine sports medicine. 2.ed. St Louis: Saunders / Elsevier, 2014. chap.8, p.108-124.

KOHN, C.; ALLEN, A.K.; HARRIS, P. Nutrition for the equine athlete. Equine Athlete, v.9, p.12-17, 1996.

LEWIS, L.D. Nutrição clínica equina: alimentação e cuidados dos equinos para desempenho atlético. São Paulo: Roca, 2000. p.293-348.
LORBIECKI, J.; KWON, S.Y.; HAMER, E. et al. Lethal Avocado Toxicity in Three Horses in North America. In: ANNUAL CONVENTION OF AMERICAN ASSOCIATION EQUINE PRACTITIONERS, 59., 2013, Nashville. Proceedings... Nashville: AAEP, 2013. v.59, p.273.

MATTOS, F.; ARAÚJO, K.V.; LEITE, G.G.; GOULART, H.D.M. Uso de óleo na dieta de equinos submetidos ao exercício. Rev. Bras. Zootec., v.35, p.1373-1380, 2006.

McCUTCHEON, L.J.; GEOR, R.J. Thermoregulation and exercise-associated heat stress. In: HINCHCLIFF, K.W.; GEOR, R.J.; KANEPS, A.J. Equine exercise physiology: the science of exercise in the athletic horse. Philadelphia: Elsevier, 2008. p.382-386.

McKENZIE, R.; BROWN, O. Avocado (Persea americana) poisoning of horses. Aust. Vet. J., v.68, p.77-78, 1991.

NADEL, E.R. Temperature regulation and prolonged exercise. In: GISOLFI, C.V.; LAMB D.R.; NADEL, E.R. Perspectives in exercise science and sports medicine. Exercise, heat, and thermoregulation. Dubuque: Brown, 1988. p.125-146.

NEGRO, S.; BARTOLOMÉ, E.; MOLINA, A. et al. Stress level effects on sport performance during trotting races in Spanish Trotter Horses. Res. Vet. Sci., v.118, p.86-90, 2018.

National Research Council. Nutrient Requirements of Horses: Sixth Revised Edition. Washington, DC: The National Academies Press. 2007. https://doi.org/10.17226/11653.

OLDHAM, S.L.; POTTER, G.D.; EVANS, J.W. et al. Storage and mobilization of muscle glycogen in exercising horses fed a fatsupplemented diet. J. Equine Vet. Sci., v.10, p.358-359, 1990.

PÖSÖ, A.R.; HYYPPÄ, S.; GEOR, R.J. Metabolic responses to exercise and training. In: HINCHCLIFF, K.W.; KANEPS, A.J.; GEOR, R.J. Equine sports medicine and surgery. Philadelphia: Saunders Elsevier, 2004. p.771792. 
R CORE team: a language and environment for statistical computing. Vienna: R Foundation for Statistical Computing, 2018. Available in: <http://www.R- project.org>. Accessed in: 20/11/2018.

RANADE, S.S.; THIAGARAJAN, P.A Review on persea americana mill (avocado) - its fruits and oil. Int. J. Pharm. Tech Res, v.8, p.72-77, 2015.

SALA, L.C.C.; ELUI, M.C.; JARDIM, M.C. Avaliação termográfica da musculatura pélvica de equinos da modalidade esportiva de três tambores. PUBVET, v.6, p.1436-1442, 2012.

SCOTT, B.D.; POTTER, G.D.; GREENE, L.W.; VOGELSANG, M.M.; ANDERSON, J.G. Efficacy of a fat-supplemented diet to reduce thermal stress in exercising thoroughbred horses. In: EQUINE NUTRITION PHYSIOLOGY SYMPOSIUM, 13., 1993, Florida. Proceedings... Florida: Equine Nutrition and Physiology Society, 1993. p.66-71.
SOROKO, M.; HOWELL, K.; ZWYRZYKOWSKA, A. et al. Maximum eye temperature in the assessment of training in racehorses: correlations with salivary cortisol concentration, rectal temperature, and heart rate. J. Equine Vet. Sci., v.45, p.39-45, 2016.

TURNER, T.A. Diagnostic thermography. Vet. Clin. N. Am. Equine Pract., v.17, p.95-113, 2001.

TURNER, T.A. Thermography as an aid to the clinical lameness evaluation. Vet. Clin. N. Am. Equine Pract., v.7, p.311-337, 1991. 